\title{
Reflection of a Wave by a Cylindrical Mirror
}

\author{
(Received 6th May, 1952.)
}

\author{
By Ll. G. Chambers
}

\section{Introduction.}

The question of the reflection of a wave by a cylindrical mirror is of interest in a number of fields. It is a problem in which it is difficult to obtain an expression for the reflected or scattered field without recourse to physical assumptions which are sometimes somewhat dubious. An attempt was made by Sommerfeld to solve the problem of a plane wave incident upon such a perfectly conducting mirror by means of what he termed the "Non-Final Determination of Coefficients". Unfortunately, a close examination of the problem renders it doubtful whether the method can be legitimately employed. It is possible, however, to solve the problem by expressing the scattered field in terms of the currents produced in the mirror, and finding the current generated in the mirror by an arbitrary incident field. The problem which we shall consider is the following twodimensional one.

We take cylindrical polar co-ordinates $(\rho, \phi, z)$ and consider a perfectly conducting infinitely thin mirror of radius $a$, whose position is defined by

$$
-\alpha<\phi<\alpha, \quad \rho=a,
$$

and a wave incident on it $\left(E_{z}=\psi\right)$ where

$$
\psi=e^{i k x}=e^{i k_{p} \cos \phi} \text {. }
$$

(We assume a time factor $e^{i \omega t}$.)

Then currents will be set up in the mirror, and these will generate what is termed the scattered, reflected or diffracted field.

\section{Sommerfeld's solution.}

Sommerfeld's method of "Non-Final Determination of Coefficients" is based on the following reasoning. (Full details ${ }^{1}$ may be had in the original

1 A. Sommerfeld, Partial Differential Equations in Physics, Ch. I, 29-31 and Ch. IV, 159-162. I have been unable to trace the original paper. 
work.) A set of coefficients $C_{n}$ is to be found which obeys the relations

$$
\begin{aligned}
\sum_{n=0}^{\infty} C_{n} \cos n \phi-f(\phi)=0, & 0 \leqslant|\phi|<\alpha, \\
\sum_{n=0}^{\infty} C_{n} \gamma_{n} \cos n \phi=0, & \alpha<|\phi| \leqslant \pi,
\end{aligned}
$$

where the $\gamma_{n}$ and $f(\phi)$ are known. Sommerfeld then states that an approximation to the solution is given by finding the minimum of the quantity

$$
E=\int_{0}^{\alpha}\left\{\sum_{n=0}^{N} C_{n} \cos n \phi-f(\phi)\right\}^{2} d \phi+\int_{\alpha}^{\pi}\left\{\sum_{n=0}^{N} C_{n} \gamma_{n} \cos n \phi_{j}^{2} d \phi\right.
$$

Clearly if $N$ is infinite $E$ vanishes when the $C_{n}$ have their appropriate values, and so $E$ may be regarded in a sense as the error of a solution.

Writing $\partial E / \partial C_{n}=0,0 \leqslant n \leqslant N$, we obtain $N+1$ equations which can be solved to give approximations to the $C_{n}$.

Close examination of this method shows that it is inadmissible for several reasons. First, there is no reason why the error of the solution should be represented by the quantity $E$ just mentioned.

It would be equally possible for the error of the solution to be represented by an expression

$$
\int_{0}^{\alpha}\left\{f(\phi)-\sum_{n=0}^{N} C_{n} \cos n\right\rangle_{\}}^{2} d \phi+k^{2} \int_{\alpha}^{\pi}\left\{\sum_{n=0}^{N} C_{n} \gamma_{n} \cos n \phi^{2} d \phi\right.
$$

which also would vanish if $N$ were infinite and the $C_{n}$ had their appropriate values. This would lead to equations for the $C_{m}$ as indicated above, with the quantities $\gamma_{n}$ replaced by quantities $\sigma_{n}=k \gamma_{n}$. The solution is thus not unique.

Secondly, if $h(x)$ is the error over $a<x<b$, and it is complex, then the quantity which ought to be minimised is $\int_{a}^{b}|h(x)|^{2} d x$. We note that $\gamma_{n}$ is complex, and so the results are untrue on this basis also.

Thirdly, whilst $\sum_{n=0}^{N} \epsilon_{n} g_{n} \cos n \phi$ is said to be the best approximation to $g(\phi)$ of the form $\sum_{n=0}^{N} \epsilon_{n} g_{n} \cos n \phi$, this is only true over the range $-\pi<\phi<\pi$, and is not true for any other range $\beta<\phi<\gamma$ where $-\pi<\beta<\gamma<\pi$. This becomes obvious if we consider the case

where

$$
g(\phi)=1 \quad\left(0 \leqslant|\phi|<\frac{1}{2} \pi\right), \quad g(\phi)=0 \quad\left(\frac{1}{2} \pi<|\phi|<\pi\right)
$$

$$
g(\phi)=\frac{1}{2}+\sum_{n=1}^{\infty} \frac{1}{\pi} \frac{\sin n \pi / 2}{n} \cos n \phi \text {. }
$$


For if the representation were to be best over the range $-\frac{1}{2} \pi<\phi<\frac{1}{2} \pi$, $g(\phi)$ would be represented to one term by $\frac{1}{2}$, which is absurd.

Thus it is clear that Sommerfeld's solution is not legitimate.

\section{Solution of the problem.}

It is frequently convenient in problems of this character to formulate the problem in terms of an appropriate Green's function. In general, if $\psi$ satisfies the wave equation inside a surface $S$, then

$$
\psi=\int_{S}\left[G \frac{\partial \psi}{\partial n^{\prime}}-\frac{\partial G}{\partial n^{\prime}} \psi\right] d S^{\prime}
$$

where $G$ is the appropriate Green's function and $n$ the outward-drawn normal. For a two dimensional problem

$$
\begin{aligned}
G & =\frac{1}{4} i H_{0}^{(1)}\left[k\left|\vec{\rho}-\vec{\rho}^{\prime}\right|\right] \\
& =\frac{1}{4} i \sum_{n=0}^{\infty} \epsilon_{n} J_{n}(k \rho) H_{n}^{(1)}\left(k \rho^{\prime}\right) \cos n\left(\phi-\phi^{\prime}\right), \quad \rho<\rho^{\prime} \\
& =\frac{1}{4} i \sum_{n=0}^{\infty} \epsilon_{n} J_{n}\left(k \rho^{\prime}\right) H_{n}^{(1)}(k \rho) \cos n\left(\phi-\phi^{\prime}\right), \quad \rho>\rho^{\prime} .
\end{aligned}
$$

Let us consider the contour indicated in Fig. 2. The integral over the large circle is equivalent to the incident field, the integrals over the straight parts cancel out, and $\psi$ vanishes over the arc of the small circle. (The tangential electric field vanishes at a conductor.) It follows that $\psi=\psi_{i}+\psi_{s}$, where

$$
\psi_{s}=\int_{-\alpha}^{\alpha} a d \phi^{\prime} . G\left(\rho, a ; \phi, \phi^{\prime}\right)\left\{\left[\frac{\partial \psi}{\partial \rho}\right]_{\rho=a+}+\left[\frac{\partial \psi}{\partial \rho}\right]_{\rho=a-}\right\} .
$$

Now it is obvious that in $\rho>a$ the scattered field will be of the form

$$
\psi_{s}{ }^{+}=\sum_{n=0}^{\infty} \epsilon_{n} \psi_{n}^{s} J_{n}(k a) H_{n}^{(1)}(k \rho) \cos n \phi
$$

and in $\rho<a$ of the form

$$
\psi_{s}^{-}=\sum_{n=0}^{\infty} \epsilon_{n} \psi_{n}^{s} J_{n}(k \rho) H_{n}^{(1)}(k a) \cos n \phi,
$$

where it has been assumed that $\psi_{i}$ is of the form

$$
\psi_{i}=\sum_{n=0}^{\infty} \epsilon_{n} \psi_{n}^{i} J_{n}(k \rho) \cos n \phi
$$


(By including only terms in $J_{n}$ we have assumed that all sources are at infinity. This not strictly necessary, but it is convenient, as is the assumption that there is symmetry in the incident field about $O x$.)

We have therefore

$$
\begin{aligned}
\psi_{s} & =k a \int_{-\alpha}^{\alpha} G\left(\rho, a ; \phi, \phi^{\prime}\right) Q\left(\phi^{\prime}\right) d \phi^{\prime}, \\
Q\left(\phi^{\prime}\right) & =\frac{1}{k}\left\{\left[\frac{\partial \psi}{\partial \rho}\right]_{\rho=a+}+\left[\frac{\partial \psi}{\partial \rho}\right]_{\rho=a-}\right\} \\
& =\frac{1}{k}\left\{2\left[\frac{\partial \psi_{i}}{\partial \rho}\right]_{\rho=a}+\left[\frac{\partial \psi_{s}^{+}}{\partial \rho}\right]_{\rho=a}+\left[\frac{\partial \psi_{s}^{-}}{\partial \rho}\right]_{\rho=a}\right\} \\
& =\sum_{m=0}^{\infty} \epsilon_{m} \cos m \phi Q_{m},
\end{aligned}
$$

where

$$
Q_{m}=2 \psi_{m}^{i} J_{m}^{\prime}(k a)+\psi_{m}{ }^{s}\left[J_{m}(k a) H_{m}^{(1) \prime}(k a)+J_{m}{ }^{\prime}(k a) H_{m}^{(1)}(k a)\right] .
$$

Hence, in $\rho>a$

$$
\begin{aligned}
\psi_{s} & =\psi_{s}^{+}=\sum_{n=0}^{\infty} \epsilon_{n} J_{n}(k a) H_{n}^{(1)}(k \rho) \cos n \phi \psi_{n}^{s} \\
& =\frac{1}{4} i k a \int_{-\alpha}^{\alpha} \sum_{n=0}^{\infty} \epsilon_{n} J_{n}(k a) H_{n}^{(1)}(k \rho) \cos n\left(\phi-\phi^{\prime}\right) \sum_{m=0}^{\infty} \epsilon_{m} \cos m \phi^{\prime} Q_{m} d \phi^{\prime} \\
& =\frac{1}{4} i k a \sum_{n=0}^{\infty} \epsilon_{n} J_{n}(k a) H_{n}^{(1)}(k \rho) \cos n \phi \sum_{m=0}^{\infty} \epsilon_{m} X_{m n} Q_{m},
\end{aligned}
$$

where

$$
X_{m n}(\alpha)=\int_{-\alpha}^{\alpha} \cos m \phi^{\prime} \cos n \phi^{\prime} d \phi^{\prime}=\frac{\sin (m+n) \alpha}{m+n}+\frac{\sin (m-n) \alpha}{m-n}
$$

and $\sin 0 \alpha / 0$ is equal to $\alpha$.

Now equating expressions for $\psi_{s}^{+}$and taking terms in $\cos n \phi$ we have

i.e.

$$
\psi_{n}^{s}=\frac{1}{4} i k a \sum_{m=0}^{\infty} \epsilon_{m} X_{m n} Q_{m}=\gamma^{-1} \sum_{m=0}^{\infty} \epsilon_{m} X_{m n} Q_{m},
$$

$\gamma \psi_{n}^{s}=\sum_{m=0}^{\infty} \epsilon_{m} X_{m n}(\alpha)\left[2 \psi_{m}^{i} J_{m}^{\prime}(k a)+\psi_{m}^{s}\left\{H_{m}^{(1)^{\prime}}(k a) J_{m}(k a)+H_{m}^{(1)}(k a) J_{m}^{\prime}(k a)\right\}\right]$.

If now we cut this short at $m=n=N$ we get $N+1$ equations for the $N+1$ unknowns $\psi_{m}{ }^{s}, 0 \leqslant m \leqslant N$, viz.

$$
\begin{aligned}
& \sum_{m=0}^{N} \psi_{m}^{s}\left\{\gamma \delta_{m n}-\epsilon_{m} X_{m n}(\alpha)\left[H_{m}^{(1)^{\prime}}(k a) J_{m}(k a)+H_{m}^{(1)}(k a) J_{m}{ }^{\prime}(k a)\right]\right\} \\
&=\sum_{n=0}^{N} 2 \epsilon_{m} X_{m n}(\alpha) \psi_{m}{ }^{i} J_{m}{ }^{\prime}(k a), \quad 0 \leqslant n \leqslant N .
\end{aligned}
$$




\section{Discussion.}

The formulae given above provide us with $N+1$ equations for the $N+1$ coefficients. Whilst the equations are too complex to enable us to obtain any general solution for the $Q_{n}$, it is however possible to derive certain conclusions from them.

In the first place, it may be seen that if we put $\alpha=\pi$, which is equivalent to taking a complete cylinder, the value of $\psi_{n}^{s}$ tends to that for scattering from a complete cylinder, viz., $-\psi_{n}{ }^{i} / H_{n}^{(1)}(k a)$.

Secondly, if $\alpha$ is very small it will be seen that the equations are of the form

$$
\sum_{m=0}^{N} \psi_{m}{ }^{s}\left\{\gamma \delta_{m n}+\text { terms } O(\alpha)\right\}=\sum_{m=0}^{N} \text { terms } O(\alpha)
$$

Hence in general $\psi_{m}=O(\alpha)$ for $\alpha$ small.

We note also that the method could be applied to consider the case of $\psi=H_{z}$ incident on the cylindrical arc. In this case $\psi$ would be given by

$$
-k a \int_{-\alpha}^{\alpha}\{P(a+)+P(a-)\} d \phi^{\prime},
$$

where $P(\rho)=\partial G / \partial \rho^{\prime} \cdot \psi$ as $\partial \psi / \partial n=0$ on metal.

The procedure would be similar to that already followed. In this case, the problem is formally identical with that of a plane acoustic wave with velocity potential $\psi$ incident upon a rigid plate.

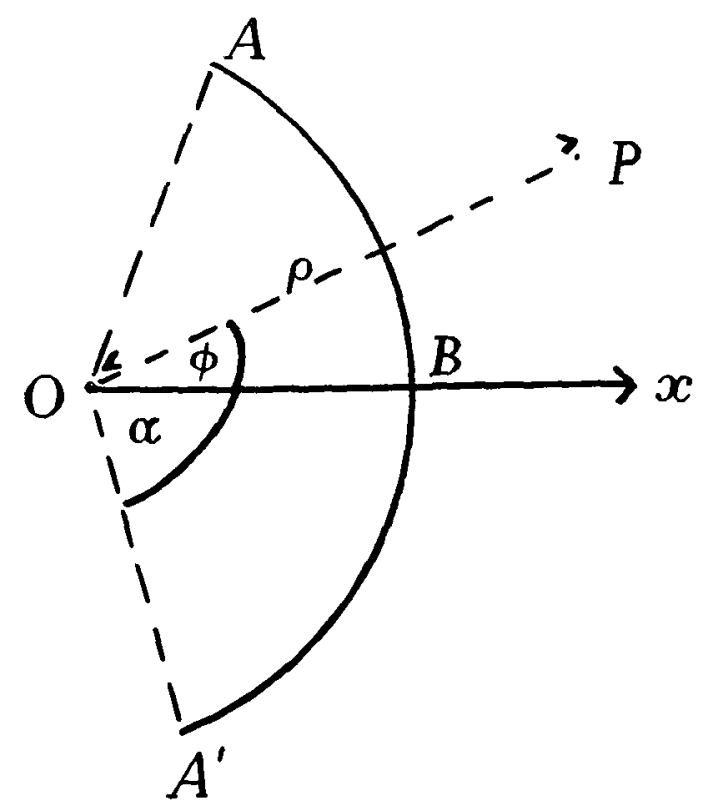

lig. 1. 


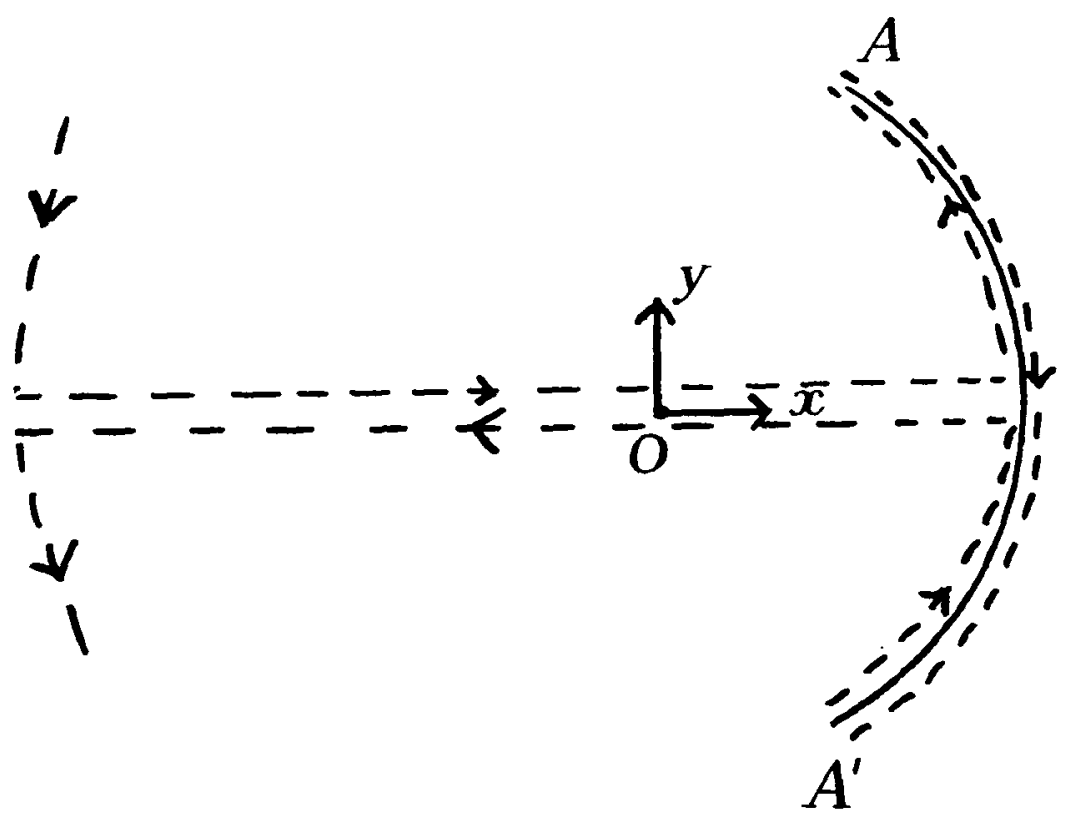

Fig. 2.

5. Acknowledgment.

This paper was written while the author was on the staff of the Royal Military College of Science, Shrivenham, and was communicated by kind permission of the Commandant.

University College of North Wales, BANGOR. 